\title{
Ueber den Entstehungsort des Lichtstaubes, der Starrblindheit und der Nachbilder.
}

Zngleich ein Beitrag;

zar Lehre von der chronischen Tabaksvergiftung.

Von

Dr. Wilhelm Filehne, Professor der Armeimittellehre in Erlangen.

Für den „Lichtstaub” oder das „Lichtchaos des dunklen Gesichtsfeldes" hat Helmholtz*) den Ausdruck "Figenlicht der Retina" eingeführt. Auch Fick**) (in Hermann's Handbuch) definirt "Eigenliclit der Netzhaut” so, dass dieser Ausdruck synonym mit. „Lichtstaub” und "Lichtchaos" ist. Wer, wie manche Ophthalmologen, unter „Eigenlicht der Netzhaut” jenes schwache, lavendelgraue Dämmerlicht versteht, welches von manchen Menschen schon nach kurzem Aufenthalt im Dunkeln, von anderen erst nach mehrstündigem Schlafe oder nie im dunklen Gesichtsfelde wahrgenommen wird, muss den "Lichtstaub" als die für die Gesichtsempfindungen wichtigere Erseheinung von dem „Eigenlicht” trennen.

\%) Fhysiol. Optik, 1867, S. 201.

**) Hermann's Handbuch, Sinnesorgane, I. Theil, S. 229.

v. Graefe's Arehiv fiir Ophthalmologie, XXXI. 2. 
Wie schon der promisene von Helmholtz für "Lichtchaos" gebrauchte Ausdruck "Eigenlicht der Netzhaut" erkennen lässt, vermuthet dieser Forscher die Ursache der Erscheinung in der Netzhaut selber.

Seite 357,1 . c. heisst es z. B.: \#. . . weil es nämlich in Wirklichkeit kein absolut dunkles Gesichtsfeld giebt, vielmehr auch bei vollständigem Ausschluss alles äusseren Lichtes doch immer noch eine gewisse schwache Reizung der Netzhaut durch innere Einflitsse bestehen bleibt, welche das schon in $\$ 17$ erwähnte Lichtchaos oder Eigenlicht des dunkeln Gesichtsfeldes hervorbringt:"

Aubert*) lässt in seiner "Physiologie der Netzhaut" die Frage nach dem Entstehungsorte des Lichtchaos unentschieden.

Fick (1. c.) vermathet, dass die Entstehungsursache in zelligen Elementen, sei es der Retina, sei es des Hirns, gelegen, und auf eine chemische Einwirkung des Blutes zurückzuführen sei. Dafür, dass es zellige Elemente des Hirns seien, scheine - seiner Meinung nach - die Thatsache zu sprechen, dass ein den Opticus aufsteigend durchfliessender Strom das Eigenlicht verstärke (beziehungsweise ein absteigender Strom es abschwäehe). Beim „aufsteigenden" Strome seien aber die centralen Parthien als im Katelektronus (erhöhter Erregbarkeit) befindlich, geeignet, das Eigenlicht stärker erscheinen zu lassen und deshalb wie hier die Stätte der Verstärkung so sonst die Stätte des Entstehens. Fick stellt dies aber nur als eine Betrachtungsweise, nicht wie einen Beweis hin. In der That liegt ein Beweis gewiss nicht vor. Zunächst ist es misslich, ron einem ,aufsteigenden Strome im Opticus" zu sprechen, wenn man am unversehrten Menschen die Anode auf die Stirn oder die geschlossenen Augenlider (die Kathode im Nacken) setzt; ebenso miss-

*) Aubert, Physiol. der Netzh., Breslau 1865, S. 336. 
lich ist es, den Elektrotonus des Opticus zu discutiren, als $o b$ nur er und zwar durch zwei ihm direct angelegte Elektroden gereizt wäre, während doch die empfindlichere Retina viel stärker vom Strome getroffen wird.

Sodann wird voransgesetzt, dass die Hirnganglienzellen die von Pflüger für die Nervenstämme ermittelten Phasen des Elektrotonus in gleicher Weise und gleichem Sinne zeigen; aber über einen Katelektrotonus der Hirnzellen mit gesteigerter Erregbarkeit und Reizerzeugung wissen wir so gut wie nichts; ja, es könnte hier im Gegensatz zum Nerven umgekehrt (und Hitzig's Reizversuche am Hirn könnten hierfür erwähnt werden), gerade im Gebiete der Kathode die Erregbarkeit der Hirnzellen vermindert und an der Anode erhöht sein. Und drittens würde, wenn wir alle jene unerwiesenen Voraussetzungen als bewiesen und richtig annehmen, die gleiche Erscheinung (Verstärkung durch aufsteigenden Strom) auch dann sich zeigen können, wenn das Eigenlicht in der Retina selber entstünde. Es brauchte nur (was dann sicherlich auch der Fall wäre), die katelektrotonische Steigerung der Erregbarkeit des Hirns und des centralen Opticus bedeutender zu sein, als die Verminderung der Erregbarkeit in den peripherischeren Theilen des Opticus durch den Anelektrotonus; alsdann würden die wesentlich empfindlicher gewordenen Centraltheile die ron der Peripherie kommenden nur wenig abgeschwächten Erregungen, welche das Eigenlicht erzeugen, mit stärkerer Empfindung beantworten. Also auch dann wäre die periphere Natur des "Lichtstaubes" nicht widerlegt.

Die Lichterscheinungen von Personen, denen ein oder beide Augen enucleirt sind, oder die eine durch Desorganisation des Opticus veranlasste Amaurose haben, sind nicht für die centrale Natur des Eigenlichts anzuführen, da nach Helmholtz (welcher das Eigenlicht peripher entstehen lïsst) hieraus nur hervorgeht (pag. 202), ,dass nicht bloss 
die Netzhaut, sondern auch der Stamm und die Wurzeln des Sehnerven im Gehirn fähig sind, in Folge von Reizungen, Lichtempfindungen zu erzeugen." Da nun das Fehlen von derartigen Reizungen in den angezogenen Fällen jedenfalls nicht bewiesen werden kann, so ist die centrale Natur des Eigenlichtes auch so nicht bewiesen. Ein wirklicher Beweis scheint mir überhaupt weder für die centrale noch die retinale Entstehung vorzuliegen.

Die andere Erscheinung, die uns beschäftigen soll, ist die „Starrblindheit". Wir verstehen hierunter die völlige Verdunkelung des Gesichtsfeldes beim längeren Hinstarren auf einen Punkt, einen Zustand, von dem wohl viele zuerst an sich selbst bei photographischer Sitzung Kunde erhielten.

Helmholtz (l. c. S. 364) erwähnt diese Erscheinung als Analogie zur Frklärung des Hinschwindens positiver Nachbilder ohne Verwandlung in negative. Er sagt: „Uebrigens schwinden auch schwache objective Bilder zuweilen in ähnlicher Weise, wenn man starr einen Punkt fixirt, z. B. eine Landschaft in der Nacht betrachtet." Eine Erklärung dieses Phänomens kann man 1. c. S. 389 entnehmen, wo rom successiven Contraste, speciell jener Form gesprochen wird, welche einen einfachen simultanen Contrast vortäuschen könne. Es wird betont, dass bei längerem Fixiren negative Nachbilder präparirt werden, ,die, so lange der Blick festgehalten wird, mit den $0 \mathrm{~b}$ jecten zusammenfallen und diese deshalb schnell undentlich werden lassen.". Hiernach wäre für Helmholtz dio Ursache der "Starblindheit", wie wir die in Rede stehende Erscheinung kurzweg nemnen wollen, dort gelegen, wo die negativen Nachbilder zu Stande kommen: also an der Stelle des Eigenlichts der Netzhaut, d. i. für ihn in der Netzhaut selber. Offenbar aber vermuthet er auch das Mitwirken eines im Bewusstsein gelegenen Moments oder, wie Hering sagt, eines spiritualistischen Moments. Denn S. 364 - hinter der zuerst citirten Stelle (s. 0.), 
fährt er fort: „Es macht mir den Eindruck, als ob die Vergleichung der Erregungsstärke verschiedener Netzhauttheile aufhörte möglich zu sein, wenn nicht die Erregung von Zeit zu Zeit wechselt."

Aubert (1. c. S. 97-105) beschreibt und bespricht diese Erscheinung ausführlich, erörtert aber die Stätte jhrer Entstehung nicht.

Die Starrblindheit wird gelegentlich gestreift von J. von Kries*) in seiner Abhandlung "Ueber Ermüdung des Sehnerven". In der Arbeit selber localisirt er die Starrblindheit nicht, die er nur bei sehr geringen Beleuchtungsintensitäten beobachtete. Er fasst sie als eine Ermüdungserscheinung auf. Gegen die auf diese Weise (durch Ermüdung) verminderte Helligkeits-Intensitat der Lichtempfindung komme bei geringer (objectiver) Beleuchtungs-Intensität das Eigenlicht der Netzbaut schon sehr in Betracht und daher könne die Reizung der sehr ermüdeten Stelle durch das schwache Licht „um so mehr unter der Grenze der Merklichkeit liegen", ,als die Unterschieds-Empfindlichkeit bei so geringen Helligkeiten überhaupt gering ist". Wo aber die Ermüdung anfasst, ob in der Netzhaut, im Opticus oder Hirn, wird erst in einem Nachtrage (S. 42-43) vorsichtig berührt: dort wird auf Grund der inzwischen erschienenen Arbeiten von Boll

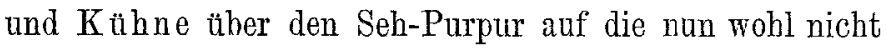
unplausible Vorstellung hingewiesen, dass ErmüdungsVerbrauch and Frholung in diesem photochemischen Materiale zu suchen sein möchten.

Was zunächst bezüglich der Starrblindheit meine eigenen Beobachtungen betrifft, so ist Folgendes etwa zu erwähnen. Bei geeigneten Vorkehrungen und einiger Uebung tritt für mich diese Erscheinung bei jeder Beleuch-

*) Dieses Arch., Jahrgang XXIIJ. Abtheil. II. (1877) S. $1-43$. 
tung und viel sehneller und plötzlicher ein, als man wach den bisherigen Angaben meinen sollte. Nothwendig ist eine bequeme Festlegung des ganzen Körpers, am besten halb. sitzend, halb liegend and hauptsächlich Jebung im Fixiren des Blickes; der fixirte Punkt liege in bequemer Sehweite. Bei schwacher Beleuchtung in minimo schon nach etwa 5 bis 10 , bei stärkerer in etwa 15 bis 25 Sekunden, bei hellster diffuser Tagesbelenchtung in etwa 20 bis 40 Sekunden - im Sonnenlichte wieder früher (z. B. etwa .15 bis 25 Sekunden) tritt (bei mir) die Starrblindheit und zwar ziemlich plötzlich und im ganzen Sehfelde wenigstens im Gebiete deutlichsten Sehens - zu gleicher Zeit ein.

Ohne jetzt schon einer Erklärung der Erscheinung nüher treten zu wollen, mache ich darauf aufmerksam, dass also die Erblindung innerhalb weiter Grenzen um so später auftritt, je, heller die Beleuchtung ist (erst bei „,blendendem" Lichte tritt sie wieder leichter auf). Innerhalb jener weiten Grenzen ist ein Widersprüch zu den Befunden v. Kries' zu sehen, sobald man geneigt ist, das Erblinden als eine Ermüdungserscheinung aufzufassen. Hier, wie bei v. Kries, ist das ermüdende Licht hinterher anch das prüfende (reagirende) Licht. Nun hat v. Kries gerade für die Ermüdung innerhalb jener weiten Grenzen der Lichtintensititen gefunden (und das stimmt mit der alltäglichen Frfahrung überein) dass die Ermüdung mit zunehmender Beleuchtungs-Intensität (übrigens nicht proportional) schneller sich entwickle; ihre Curve ist aber zur (Zeit-)Abscisse convex und die Empfindungsgrösse wird bei v. Kries - abgesehen von den erwähnten Versuchen mit minimaler Beleuchtung - niemals (selbst nach mehr als zwei Minuten nicht) Null. In unseren Verstchen aber wird die Empfindungsgrösse überall Null und namentlich wird sie es um so später, je heller die Beleuchtung. Nun ist aber ja offenbar die Starrblindkeit bei mini- 
maler, bei mittlerer und starker Beleuchtung überall die gleiche Erscheinung. So ist also zu vermuthen, dass beim starren Fixiren neben der "Ermüdung" von Kries' noch etwas anderes Platz greift, was sich diesem Antor bei minimaler Beleuchtung zwar aufdrängte, weil es dort sehr leicht und schnell eintritt, was aber bei seinen übrigen Beobachtungen nicht zur Entwickelung gebracht wurde; dann wird aber die Zulässigkeit seiner Auffassung von der bei schwacher Beleuchtung auftretenden Starrblindheit zum mindesten in Frage gestellt.

Während der Starrblindheit sieht man - was nirgends hervorgehoben zu sein scheint - nicht etwa "nichts" oder "schwarz", sondern normales Lichtchaos, wie im absolut finstern Raume.

Wird nun ein im beobachteten bellen Raume vor lichtlosem Grunde gesehener weisser Gegenstand - z. B. die eigene Hand - während der Blindheit plötzlich entfernt, so entwickelt sich - gleichviel ob die Hand von der Fixirlinie getroffen wurde oder nicht - Folgendes: Es verschwindet aus dem lichtchaotischen Gesichtsfelde an jener Stelle, welche das Bild des beseitigten Gegenstandes hätte einnehmen sollen, der Lichtstaub ganz (oder fast ganz) und ein tief schwarzes, genaues Bild (z. B. der Hand mit ausgespreizten Fingern) erscheint. Das Eigenlicht der Umgebung leuchtet auf. Bild und Lichthof klingen dann bei fortgesetztem Fixiren zum normalen Lichtchaos wieder ab.

(Wenn während der Starrblindheit die beobachtete Räumlichkeit objectiv verdunkelt wird, oder der Beobachter die Augen schliesst, erscheint, minder deutlich zwar, ein Negativ des vor dem Eintritt der Blindheit gesehenen Gesammtbildes.)

Wodurch entsteht die Starrblindheit? Und wo ist die Verbindung zwischen Lichtquelle und Bewusstsein unterbrochen? 
Dr. Wilhelm Filehne.

Die Entstehungstätte dieser Blindheit könnte in der direct gegen Licht empfindlichen Retinaschicht, oder zweitens in der Leitung irgend wo, oder drittens im Hirn gelegen sein.

Nehmen wir zunächst an, die Ursache dieser Blindheit läge in der Peripherie. Dies würde bedeuten, dass bei dieser Lichtintensität, z. B. für Weiss, das photochemische Material aufgebraucht sei und dass nunmehr (nach Hering) keine Dissimilation und deshalb keine Erregung mehr zu Stande komme. Direct widerlegt wird diese Anffassung aber dadurch, dass nach Entfernung des weissen Objects im Lichtchaos ein schwarzes, scharfes Bild entsteht. Wenn während der Starrblindheit keine Dissimilation mehr stattfände, keine Erregung der lichtempfindlichen Sehicht - dann müsste es gleichgiltig sein, ob der weisse Gegenstand dort bleibt oder nicht. Es könnte später nicht eine so heftige Assimilationserregung (Empfindung des Schwarz) auftreten und es könnte nicht in ihrer Nachbarschaft eine durch das Aufleuchten eines lichtchaotisehen Hofes markirte dissimilatorische Erregung erzeugt werden. Hieraus folgt, dass wir vorher, während der Starrblindheit, blind gewesen sind, obwohl die lichtempfindliche Peripherie vom Lichte erregt wurde.

Wer nun zugiebt, dass die Ursache der Starrblindheit nicht in der lichtempfindlichen Peripherie gelegen ist, wird kaum im Zweifel sein, dass sie dann in der Ieitung bis zum Gehirn nicht, sondern nur im Hirne selber sich befinden könne. Die Gleichzeitigkeit und verhältnissmässige Plötzlichkeit des Erblindens im ganzen Felde setzt entweder eine innige physiologische Verbindung der betheiligten Gewebselemente, wie im Netze der Nervenzellen des Hirns - oder eine überall gleichartig einwirkende übermächtige äussere Kraft bei gleicher Widerstandsfähigkeit (oder Widerstandsunfähigkeit) voraus, wie dies in der gegen Licht direct empfindlichen Peripherie 
bei ihrer durchgehends gleichen Ernährung a priori allenfalls zuzugeben wäre. Für die von sinander physiologisch isolirten und verschieden in Anspruch genommenen Leitungsorgane treffen beide Bedingungen nicht zu. Jedenfalls aber, glaube ich, ist schon jetzt der Beweis geliefert, dass in der Peripherie die Ursache der Starrblindheit nich $t$ zu suchen ist.

Welcher Art kann aber dieses Hinderniss sein? Eine einfache "Ermüdung" im Sinne des gewöhnlichen Sprachgebrauchs ist deswegen zurückzuweisen, weil - z. B. bei Fixirung eines Holzgetäfels oder eines Gypsreliefs und anderer gleichfarbiger Dinge - in der Starrblindheit eine kleine Verrückung des Objects, des Blicks oder des Gesammtkörpers bei gleichbleibendem Blicke genügt, um wieder Alles zu sehen; dies zeigt, dass eine angeblich ermüdete Stelle sofort wieder die lichtempfindung vermittelt, wenn ein z. B. schwächerer Impuls von der Peripherie her ihr zugeführt wird; nur gegen diesen bisherigen ist das Sehorgan ,abgestumpft" - jeder andere, schwächere oder stärkere erregt es wieder. Will man aus dem Sprachschatze ein Wort hierfür wählen - was ja keine Erklärung, wohl aber einen Analogien-Complex liefern kann so ist dies keine Ermüdung, sondern gehört in das Gebiet der Gewöhnung. Wir lätten es daher bei der Starrblindheit mit dem Aufhören von Empfindung, trotz fortdauernd zugeleiteter Erregung in Folge ron Gewöhnung zu thun. Derartiges kommt aber nur bei Centralorganen und nicht bei Leitungsorganen vor.

Zahlreiche Analogien hierfür giebt es bekanntlich im Gebiete anderer Sinne: Die Feder hinterm Ohr, die man nicht mehr fühlt; den auffallenden Geruch, den wir beim Eintritte in einen Raum wahruehmen, spüren wir sehr bald nicht mehr; der Müller hört das Klappern der. Mühle nicht mehr; das Schnurren eines Inductions-Apparates entschwindet nach einiger Zeit dem Gehör. Hier ist auch 
die Analogie des "Schwar" nach Entfermung des "Weiss" bei Starrblindheit za erwähnen: wenn Jemand den nicht mehr gehörten Inductions-Apparat plötzlich zar Ruhe bringt, so haben wir eine positive, nicht näher beschreibbare Empfindung, die allmahlich abklingt.

Nun könnte man gegen diese Analogisirung einwenden: Der Müller kann, wenn er will, den Mühlgang, und wir, wenn wir unsere Aufmerksamkeit darauf richten, das Schnurren des Apparats jederzeit wieder wahmehmen, während die Starrblindheit durch Aufmerksamkeit nicht zul bewältigen ist. Bei Geruch und Gefuhl gilt jenes aber sehon nicht. Und einerseits giebt es auch beim Sehen solche Dinge, welche wir für gewohnlich - in Folge von centraler Reizgewöhnung - nicht wahrnehmen und die wir jederzeit, wenn wir unsere Anfmerksamkeit dorthin lenken, zur Wahrnehmung bringen können, z. B. das Lichtchaos boi halbheller Beleuchtung: Hs muss schon sonnenheller Tag sein, wenn ich das Lichtchaos nicht soll seben können, sobald ich darauf achte, und doch sehe ich es für gewöhnlich auch in Dämmerlichte noch nicht. Andererseits ist anch am Gehörorgan eine durch Beachtung nicht zu beseitigende Reizgewöhnung mehrfach zu statuiren, z. B. kö̈nnen wir den fortwährend in unseren Ohren erKlingenden Resonnanzton des Mittel-Ohres durch blosses Hinlenken der Aufmerksamkeit nicht wahrnehmen. Wir sind "tanb" gegen jenen Ton, wie wir "blind" gegen einen gleichbleibenden Gesichtseindruck werden.

Unser Experiment mit der Beseitigung eines weissen Gegenstandes während der Starrblindheit lässt sich anch verwerthen, um einiges über die Lage derjenigen physiologischen Stätte zu erschliessen, an welcher der „Lichtstaub" zu Stande kommt. Es sei beiläufig darauf hingewiesen, dass die von der lichtempfindlichen Schicht kommende assimilatorische Erregung, welche die Enpfingung voin "Schwaiz" mitten in leuchtender Finsterniss er- 
zeugt, diejenigen Erregungen übertönt, welche das „Eigenlicht" liefern. Wir wissen ferner, dass das objective Licht bei einer zur Lichtempfindung völlig hinreichenden Beleuchtung (im Halbhellen und Hellen) Erregungen in der lichtempfindlichen Schicht and weiter im Opticus veranlasst, welche stärker sind, als diejenigen, aus denen das Lichtchaos resultirt (daher wir alsdann die Aussenwelt and nicht das Lichtchaos sehen). Wo immer im nervösen SelApparate bei der Starrblindheit das blindmachende Hinderniss sich entwickeln mag, welches die stärkeren, durch intensiveres, objectives Licht bewirkten Erregungen nicht mehr passiren lïsst: die dem Lichtstaube zu Grunde liegenden schwächeren Erregungen können dort um so gewisser nicht durchdringen. Da aber in der Starrblindheit der Lichtstaub normal gesehen wird, da also diese schwachen Erregungen zu den Organen des Bewusstseins gelangen, so müssen sie entweder unmittelbar am Hinderniss oder zwischen Hinderniss und den Organen des Bewusstseins entstehen. So ist also die Entstehungsstätte des Lichtstaubes noch centraler oder wenigstens ebenso central wie die der Starrblindheit.

Der Nachweis, dass diese „centralere" Entstehungsstätte wirklich im Gehirn, nahe den Organen, welche dem Bewusstsein dienen, gelegen ist, lässt sich auf anderem Wege wirksam versuchen.

Nicht streng wäre folgende Beweisführung:

Bei genauerem Betrachten sieht man bekanntlich nicht bloss "Lichtstaub" sondern Fäden, Arabesken, Bewegung, concentrische, wandernde Nebelstreifen, wechselnde Teppichmuster, die das ganze Gesichtsfeld oder einen nennenswerthen Bruchtheil desselben gleichmässig, aber kaleidoskopisch in Farbe und Form wechselnd, bedecken, Feuerspiralen u. s. w. (von den Phantasmen ganz zu schweigen). Derartiges kann in der Retina selber mit ihren isolirten lichtpercipirenden Elementen und isolirten Leitungen nicht 
zu Stande kommen (umsomehr als nur ein Muster u. s. w. mit beiden Angen gesehen wird), es setzt dies einheitlich verbundene und centrifugal disponirende Action vorans und kann daher nur vom Hirn veranlasst werden, wäbrend ein einfacher ungeformter Lichtstaub allerdings in der Retina hätte erzengt werden können. Hieranf könnte man aber erwidern, dass sehr wohl der Lichtstaub von der Retina geliefert werden könnte, und das Gehirn nur die Muster, Bilder u. s. w. (zumal natürlich die Phantasmen, worauf schon Aubert hinwies) hineinsieht, wie man in rule Wolken, Felswande, complicirte Tapetenmuster und farbenbetupfte Blächen, aus einer gewissen Entfernung sie betrachtend, allerlei Bilder und Muster hineinsiebt. Die Gruppirung ist also selbstverständlich Hirnarbeit; ob aber der Lichtstaub, aus welehem das Hirn diese Figuren formt, nicht in der Retina entsteht, bleibt fraglich.

Dagegen seheint mir Folgendes nachdrücklichst für die centrale Entstehung des Lichtstaubes zu sprechen:

Mit einem beiderseitigen centrischen und lateralparneentrischen, scharf ausgeschnittenen Scotom, mit völliger Frblindung des beiderseitigen Fixirpunktes und seiner Umgebung, wie dies in Folge von Tabaksintoxication eine Leit lang bei mir bestand, sah ich dennoch stets ungeschwächt überall den Lichtstaub (später sogar in verstärktem Masse).

Wo auch immer die Tabaksvergiftung das blindmachende Hinderniss setzen mag, welches die stärkste Einwirkung objectiven Tichtes nicht durchlässt, jedenfalls muss die Fntstehungsstätte des ungeminderten Jiehtstaubes, der doch auf so schwachen Erregungen beruht und so leicht übertönt werden kann, oberhalb, central von jener Angriffsstelle des Nicotins liegen. Sobald daher als bewiesen gelten kann, dass die lähmende Wirkung des Nicotins beim Tabaks-Scotom die cerebralen Theile des nervösen Beh-Apparates mod nicht die Peripherie trifft, 
so ist implicite damit bewiesen, dass die Entstehungsstatte des Lichtstaubes mindestens eben so hoch in Gehirn gelegen ist.

Dass der Angriffspunkt des Tabaks im Gehirn liegt, lässt sich als höchst wahrscheinlich hinstellen. Förster*) und nach ihm Hirschberg**) machen zwar gegen seinen cerebralen Sitz geltend, dass die scomatösen Stellen der beiden Sehfelder (rechts und links) anatomisehsymmetrischen Stellen der Netzhäute, nieht aber physiologisch - identischen Partien entsprechen. Indess beweist diese Thatsache doch nur, dass der Sitz des Tabaksscotoms nicht in jenen Abschnitten des Hirns liegt, welche die beiden Sehfelder zu einem Gesichtsfeld verschmelzen; aber sie kann uns nicht hindern, die Ursache des Scotoms in einer diesseits gelegenen Projection der Netzhüute innerhalb des Gehirns, also an (vielleicht selber anatomischsymmetrischen) Stellen zu suchen, welche mit anatomisch symmetrischen Netzhautpartien (r. u. 1.) communiciren. Freilich muss dies aber erst bewiesen werden:

Der negative ophthalmoskopische Befund (bei mir von Herrn Collegen Sattler constatirt) kann nicht ausschliessen, dass in der Retina eine functionelle Stürung besteht - und mehr als functionelle Störung ist ja auch im Hirn nicht vorauszusetzen. Beweiskrüftiger ist der Habitus der Affection: die Localisation, die scharfe Abgrenzung des Scotoms an anatomisch vor der unmittelbarsten (gesundgebliebenen) Nachbarschaft in der Netzhaut nicht ausgezeichneten Stellen spricht entschieden gegen retinale und für cercbrale Angriffsstelle. Ich glaube einige neue, so viel ich erfahren habe und finden kounte, noch nicht bekannte Beobachtungen als Anzeichen für die

*) In Graefe und Sämisch, Handb. d. ges, Augenheilk., Bd. VII, S. 201.

**) Zeitsehr. f. pract. Med. 1882 No. 17 (S. 198) und No, 18 ธ. 205). 
cerebrale Natur des Tabaksscotoms beibringen zn können. Zunächst führe ich eine meiner Meinung nach nicht beweiskräftige neue Beobachtung an: Nachdem das Scotom etwa 4 Wochen bestanden hatte, wurde ich auf eine Erscheinung aufmerksam, von der ich nun nicht sagen kann, ob sie von Anfang an vorhanden war. Von Zeit zu Zeit - zuweilen in einer Minute 2-3 Mal auftretend, zuweilen während 10 Minuten und noch länger ausbleibend blitzte es bei geschlossenen Augen oder im Finstern im Scotom-Gebiete wie bei Schluss eines galvanischen Stromes auf, kein Funke oder Strahl, sondern wie Wetterlenchten, theils rom Fixirpunkt entspringend und sich über das ganze Sehfeld ausbreitend oder anch an einem Ende des Scotoms begimnend und zum anderen sich fortpflanzend. Wine solche uber einen grösseren Theil des Gesichtsfeldes sich ergiessende Lichterscheinung ohne nachweisbare äussere oder intraoculare Ursache durfte wohl mit Recht als cerebral bedingt aufgefasst werden. Aber damit ist die cerehrale Entstehung des Scotoms nicht erwiesen, welches möglicherweise doch in der Retina entstanden sein und secundär erst zu jener cerebralen Lichterregung Veranlassung geben könnte. (Dass derartige als offenbar cerebral imponirende Lichtempfindungen in Folge ron localer Retina-Affection erscheinen können, habe ich an mir selbst erfahren. Ich habe in meinem von Natur schwächeren reohten Ange in Folge eines vor einer längeren Reihe von Jahren erlittenen localen Traumas eine auch ophthalmoscopisch [Prof. Sattler] erkennibare excentrisch gelegene schmale, streifenformige blinde Stelle in einer Länge ron $1 / 5$ Durchmesser des Sehfeldes. In dieser Partie habe ich schon wenige Woehen nach jenem Trauma ein noch heute fast täglich gelegentlich nach Körperanstrengung u. s. w. sich zeigendes, damals aber alle 5 Sekunden auftretendes Phosphen wahrgenommen, welches am besten mit einem starken, niedergleitenden 
feurigen Flüssigkeitstropfen geschildert ist, der, plötzlich nach aufwäts biegend, verschwindet und im ganzen seinen Weg ron etwa $1 / 5$ Durchmesser des Sehfeldes in einer halben Sekunde zurücklegt. Trotzdem ist dieses Scotom zweifellos peripher bedingt.)

Bei meinen Tabaksscotomen machte ich femer folgende Beobachtungen. Schon seit deutlichem Beginn der Affection konnte ich bei genauerer Aufmerksankeit die Scotome beim Oeffinen nur eines Auges und bei Blinzeln, sowie bei monocularer gleichmässiger Betrachtung meines Weges während des Gehens, auf hellere Elächen projiciren. Auf sebr hellen weissen Flächen waren die fast rein elliptischen, mit ihrem Durchmesser horizontal gestellten Flecke bräunlich*). Blickte ich nach längerem Augenverschluss auf eine sehr helle Landschaft, so war zuerst auch das ubrige centralere Gesichtsfeld ganz undeutlich und wie von einem hier und da metallisch glänzenden, grünlichen und röthlichen Wolkenschleier verdeckt und erst nach 5 bis 15 Sekunden wurde der Blick klar. Bei schwacher Tagesbeleuchtung oder Gas- und Lampenlicht-sah ich, auch ohne darauf zu achten und gegen meinen Willen, alle weissen oder indifferenter gefärbten Flächen (z. B. Papier, Gesichter) beständig roth und grün marmorirt. Nur

*) Auf einfarbige Elächen projicirt, gab es eine wesentlich unregelmässiger, bei jeder Farbe besonders geformte, stellenweise durchlocherte und in sich und am Rande etwas amöboide Berregung zeigende, meist bräunliche, eine leise Spur der Contrast-Farbe tragende Wolke, durch deren Löcher hier und da der farbige Grund durchschimmerte. Dementsprechend gingen kleine, am Perimeter bewegte farbige oder auch weisse Objecte an manchen Stellen wie hinter zerrissenen Wolken. Am intensivsten, extensivsten und anbaltendsten hatte Blau gelitten; wesentlich weniger dagegen Roth und Grün. Weiss (und ebenso Gelb) war zwar in jeder Beziehung am günstigsten, verschwand gänzlich aber auf der Höhe der Affection in einem grossen Bezirke des Scotoms, welches demnach in diesem Bezirke ein absolutes war. 
selten ging das Roth in Violett über und nur dann weehselte zuweilen stellenweise auch das Grün nach Gelb hin unter Aufleuchten; war dies geschehen, dann nahm das Violett, seinerseits aufleuchtend, einen gesätigteren Farbenton an. Diese Marmorirung betraf nicht nur das ganze Scotom, sondern erstreckte sich anch noch über die Grenzen desselben hinaus. Die Marmorirung war eine in jeder Beziehung fortwährend wechselnde, so dass jeder Punkt des marmorirten Gesichtsfeldes bald grün bald roth war; die breiten rothen und grünen Farbenmassen wälzten sïch fortwährend durcheinander (iibrigens auf den beiden Augen verschieden $\left.{ }^{*}\right)$ ).

Dass eine mit letzterer Eigenthümlichkeit ausgestattete Affection nicht in der Retina ihren Ausgangspunkt haben kann, ebensowenig wie in dem. Leitungsnervenstamme, sondern cerebral bedingt sein muss, erscheint selbstverständlich. Die subjectiven simultanen Farben-Contraste denten nebenbei bémerkt - an, dass die Stätte des Contrastes und der Angriffspunkt des Tabaks plysiologisch nächste Nachbarn sind, wenn nicht identisch.

Ein anderes vielleicht interessanteres Anzeichen für die cerebrale Entstehung des Tabaks-Scotoms ist in folgender Beobachtung gelegen. In meinem von Natur besonders gut fungirenden linken Auge hatte ich in demjenigen Gebiete, welches dem blinden Fleck des anderen Auges entspricht, noch eine kleine scotomatöse Stelle inmitten gut sehender Umgebung. Die entsprechende Retina-Stelle ist bekanntlich anatomisch in keiner Weise vor ihrer Umgebung ausgezeichnet. Wohl aber ist derjenige Hirntheil, welcher mit dieser Stelle correspondirt, physiologisch ausgezeichnet, da er im Gegentheil zu seinen Nachbarn keinen

a) Dies beweist, dass die Entstehung auch dieser Erscheinung nicht in dem Organe gesucht werden darf, dessen Function die Verschmelzung der beiden Sehfelder zu einom gemeinsamen Felde ist. 
identischen Paarling hat. Sowie also der physiologisch bevorzugte Fixirpunkt beiderseits erblindet, ebenso erkrankt auch diese bevorzugte Stelle, die peripherisch nicht, wohl aber in der cerebralen Verknüpfung sich von ihrer Nachbarschaft unterscheidet. Dies spricht entschieden für cerebralen Sitz des Scotoms.

Wenn wir demnach die Angriffsstelle des Nicotins nicht in der Retina, sondern im Gehirn zu suchen haben, und wenn ich trotz meiner temporären, scharf begrenzt localisirten vollständigen (und unvollkommenen) Erblindung den Lichtstaub in normaler Erscheinungsweise überall, also auch in der auf beiden Augen erblindeten Umgebung des Fixationspunktes gesehen habe, so muss die Entstehungsstelle des Lichtstaubes wenigstens ebensoweit central wie die Angriffsstelle des Tabaks, also im Gehirn liegen.

Wo aber der Lichtstaub entsteht, da entstehen auch die Nachbilder, der Contrast und die Starrblindheit.

Indess ist deswegen für den Contrast keineswegs auf den Standpunkt der Urtheilstäuschung zurückzukehren; vielmehr wären die nicht-spiritualistischen Vorstellungen Hering's nur von der Retina anf Hirnbezirke zu übertragen.

Als ich die vorstehend gegebenen Erfahrungen und Erwägungen Herrn Collegen Aubert (Rostock) mittheilte, hatte derselbe die Güte, mich auf zwei Einwände aufmerksam zu machen: nämlich erstens, dass ein auf das Auge einwirkender mechanischer Druck jede Art von Eigenlicht beeinflusst, und zweitens auf $\mathrm{S}$. Exner's*) für die retinale Entstehung der positiven Nachbilder gegebene Beweisführung. Ueber Beides habe ich seit dem experi-

*) Repertor. d. Physik, Bd. XX, S. 374 (Protoc. der chem.physik. Ges. zn Wien, 18. März 1884).

Vergl. auch: Pflüger's Arch., Bd. XX, S. 614 ff.)

v. Graefe's Archiv für Ophthalmologie XXXI. 2. 
mentirt und glaube jetzt die cerebrale Entstehung aller genannten Erscheinungen anfrecht erhalten zu müssen.

Zunächst Exner's Beweis furr die retinale Entstehung der positiven Nachbilder: Finner bringt zwei Thatsachen: einmal das frühe Verschwinden des Nachbildes, wenn auf den Bulbus ein stärkerer. Druck ausgetibt wird; dieser Punkt wird uns nachher zugleich mit der Beeinflussung des Eigenlichtes durch Druck auf den Bulbus zu beschäftigen haben; denn diese beiden Experimente sind, wie man sieht, innerlich gleichbedentend. Sodann aber Folgendes: durch Benutzung rotirender Scheiben mit sehwarzen und weissen Sectoren sei, sagt Exner, festgestellt, dass (bei mittlerem Tageslicht) eine häufiger als 24 Mal in der Sekunde erfolgende Erregung der Retina durch objectives Licht nicht mehr discontinuirlich empfunden werde. Dass diese Trägheit der Reaction (das Festhalten des positiven Nachbildes) nicht im Hirn oder im Nervus opticus zu suchen sei, ginge daraus hervor, dass eine 60malige elektrische Reizung des Buibus noch discontinuirliche Lichtempfindong erzenge. Gehirn und Nerv können also $60 \mathrm{Mal}$ in der Reaction wechseln - also läge die Trägheit bei Reaction auf Lichtwechsel - mit anderen Worten: das positive Nachbild - in der Retina. So bestechend Manchem diese Schlussfolgerung auf den ersten Blick erscheinen mag - sie ist unrichtig, und ich kann den zweifellosen Nachweis liefern, dass das Sehorgan in seiner Totalität gegen den elektrischen Strom bezüglich dieser zeitlichen Verhältnisse genau so reagirt, wie.gegen objectives licht und damit fällt dieser scheinbare Beweis Fxner's für die retinale Natur des positiven Nachbilds.

Die gleichmässig grau werdenden rotirenden Scheiben und das subjective durch elektrische Reizung erzengte Blitzen, erschienen mir nicht reeht commensurabel: ich beschloss, das subjective elektrische Licht mit objectivem elektrisehem Lichte gleicher Helligkeit und Zeitdauer zu 
vergleichen. Die für diese, sowie die weiter zu erwähnenden Versuche nothwendigen physikalischen Apparate hat mir unser Physiker, Herr College Lommel, in liebenswürdigster Weise zur Verfügung gestellt.

Mittels eines Zahnrades, welches verhältnissmässig langgestreckte, aber sehr niedrige Zähne besitzt, kounte ich abwechselnd einerseits die Zahl der elektrischen Reizungen der Retina, andererseits die Häufigkeit der elektrisçhen Durchleuchtung einer Geissler'schen Röhre reguliren. Zur Reizung nahm ïch nicht (bloss), wie Exner, die minder zweckmässigen Inductionsströme sondern kürzeste Ausschnitte aus einem galvanischen Strome. Die Reizung fand nur beim Ueberspringen des Contactes ron einem Zahn zum andern statt, nicht während des Schleifens auf dem Zahnrücken. Von einer Batterie, welche sonst mit zwei Elementen schon starke subjective Blitze giebt, wurden so 36 Elemente nöthig. Bis 60 Reizungen in der Sekunde erschienen bei mir selbst und bei drei Personen mit gesunden Augen - in Uebereinstiminung mit Exner discontinuirlich; bei $60-75$ begann die Erscheinung continuirlich zu werden. Jetzt wurde vor die Geissler'sche Röhre ein transparenter Schirm angebracht, vor welchen sich die Versuchsperson in solcher Entfernung stellte, dass der vom Funken erzeugte Lichtschein dieselbe Intensität wie das frühere subjective Blitzen hatte. Das Zahnrad steigerte dann allmählich die Frequenz des Funkenspringens, bis der Schein auf dem Schirme continuirlich wirkte. Und wieder war es über 60 (etwa $70 \mathrm{Mal}$ ) in der Sekunde.

Wer nun, wie Exner (l. c. und Pflüger's Arch., Bd. XX, S. 614, Anmerk.), den Angriffspunkt des elektrischen Reizes nicht in der direct lichtempfindlichen Schicht, sondern in dem Anfange der Leitung sieht (was wohl richtig ist), der wird jetzt umgekehrt den Beweis für die cerebrale Natur der Fmpfindungsverschmelzung als erbracht 
ansehen. Indess genügt es mir, wenn zugegeben wird, dass Exner's scheinbarer Beweis der retinalen Natur des positiven (gleichgefârrbten) Nachbildes abgewiesen ist.

Es lag mir daran, den Unterschied zwischen der optischen Wirkung der rotirenden Scheiben und der Geissler'schen Röhre kennen zu lernen. Die vergleichenden Versuche hierüber ergaben nun Folgendes: Auch mit rotirenden Scheiben ist (bei schwacher oder mittlerer Tagesbeleuchtung), unter Berïcksichtigung sogleich zu erwähnender Versuchsbedingungen, dasselbe Unterscheidungsvermögen für discontinuirliche Reizung am (normalen und tabalssamblyopischen) Sehorgane zu constatiren, wie mit elektrischem Lichte; orst bei etwa 75 Unterbrechungen in der Sekunde wird dann die Empfindung continuirlich. Wemn Exner die Zahl von 24 Intermittenzen p. Sec. als Grenze empfindbarer Discontinuität ansieht (Plateau hat 60, Aubert die Zahl 50 angegeben), so ist diese Zahl Helmholtz's und Emsmann's für Versuche mit Scheiben, welche in zwei bis acht schwarze und weisse Sectoren getheilt sind, freilich richtig. Aber wie man sich leicht überzengen kann, steigt mit zunehmender Sectorenzahl, also abnehmendem Winkel der schwarz-weissen Periode, erst langsam, dann sehr schnell, die zur Erzeugung continuirlicher Empfindung erforderliche Zabl der Unterbrechungen. (Wir wollen diese Zahl der Kürze wegen „Intermittenzzahl" nennen.) Schon bei 16 und 24 Sectoren ist die Intermittenzzahl etwa 45 und 60 . Von da an steigt sie langsamer und erreicht bei etwa $48-60$ Sectoren ihr nun nicht mehr uberschreitbares Maximum ron etwa 75 .

Somit ist also thatsachlich kein Unterschied in den Resultaten der beiden Versuchsreihen, mit. rotirenden Scheiben einerseits und Geissler'scher Rouhre andererseits.

Mehr beiläufig sei noch Folgendes erwähnt und erortert: 
Plateau's, von Helmholtz bestätigte Angabe, dass die Zahl der zur Erzengung continuirlichen Graues nothwendigen Umdrebungen der Scheibe nicht geändert wird, wenn man in dem einen Falle schmale weisse Sectoren mit breiten schwarzen und in anderem Falle ebensoviele, aber breite weisse und schmale schwarze Sectoren wählt, zeigte sich mir für alle Intermittenzzablen giltig. Dass also gleiche Intermittenzzahl bei verschiedener Helligkeit des producirten continuirlichen Grau vorkommt, wusste schon Plateau. Aber er hatte nicht erkannt, dass bei gleicher Helligkeit des Grau (z. B. wenn auf jeder einzelnen Scheibe die weissen Sectoren ebenso gross sind, wie die schwarzen) sehr rerschiedene Intermittenzzahlen zu statuiren sind, z. B. 25 bei Quadranten and 75 bei Sectoren von je $6^{\circ}$. Nun unterscheiden sich. diese Fälle

$$
\left(25 \cdot \frac{2 \pi}{4} \text { und } 75 \cdot \frac{2 \pi}{60}\right)
$$

äusserlich nur durch die verschiedene Winkelgeschwindigkeit.

Je grösser also die Winkelgeschwin digkeit, um so mehr nimmt die Normalzahl $75 \mathrm{ab}$, zuerst langsam, dann schneller, dann wieder langsam bis zu einem unüberschreitbaren Minimum von etwa $25 \mathrm{p}$. Sec*).

Die theoretische Betrachtung wird bequemer, wenn man die Winkelbewegung in eine gradlinige verwandelt; es

*) Eine vereinzelte von Plateau (Pogg. Ann. 1830, Bd. XX, S. 315-316) angefübrte Thatsache gehört der Sache nach, obschon uicht Pla tea n's ldeengange nach, hierher, ist ubrigens weder von $\mathrm{Pl}$., noch von den späteren Autoren in unserem Sinne verwerthet worden. Man findet dort, bei achtzehn weissen "Sectoren auf der einen schwarzen Scheibe und sechs ebenso breiten weissen Sectoren auf einer anderen schwarzen Scheibe, die Intermittenzzahl für erstere grösser ale für die letztere. Plateau's Erklärung geht aber von der grösseren Helligkeit der ersteren Scheibe aus, während hier bei uns Scheiben von gleicher Helligkeit (je $180^{\circ}$ Weiss und $180^{\circ}$ Schwarz, nur in verschiedener Vertheilung) verschiedene Intermittenzzahlen geben. 
werde also der Radius der Scheibe $=\infty$; dann werden die Winkelbögen absolute Längen. Bänder, in gleichmässig wiederkehrender Strecke schwarz und weiss gefärbt, sind das Object, welches sich in gerader Linie vor dem Auge (in bestimmter Entfernung) verschiebt. Je langer die Strecken sind, um so schneller muss das Band (gleiche mittlere Beleuchtung und nicht in Frage kommen der Sehschärfe vorausgesetzt) vorübergleiten, um gleichmässiges Crau zu geben; aber je grösser die Strecken, umsomehr nimmt innerhalb gewisser Grenzen die Intermittenzzahl ab. Da die Helligkeit des resultirenden Grau's bei allen Bändern gleich ist, da auf der weissen und der schwarzen Strecke an und für sich dem Ange nichts die Geschwindigkeit verräth, so entscheidet ausschliesslich die Geschwindigkeit, mit weloher das Bild der Trennungslinie zwisehen je einer weissen und einer schwarzen Strecke sich anf der Retina bewegt. Je plötzlicher die neue Farbe erscheint, um so langsamer klingt sie an, um so langsamer die vorige ah.

Nur nebenbei sei angedeutet, dass an der Grenzlinie die simultanen Contraste bei geringerer Geschwindigkeit Zeit haben, sich zu entwickeln und dann, bei trotzdem gleichbleibender Gesammthelligkeit, die Unterscheidung der Discontinuität begünstigen.

Warum die Intermittenzzahl nicht über jenes Maximum und nicht unter ein gewisses Ninimum geht, bedarf wohl keiner Discussion*).

*) Gelegentlich kam ich bei diesen Versuchen anf eine Beobachtung, die oben im Texte nicht in Betracht kommen kann, die aber von Anderen gelegentlich vielleicht als Demonstrations-Versuch oder sonst verwerthet werden könnte und deshalb hier folge: In einer Scheibe, welche einen um 2-3 Ctm. grösseren Halbmesser als die schwarz-weissen Scheiben besitzt, wird parallel zur Peripherie und einen Centimeter von ihr entfernt ein $180^{\circ}$ betragender Schlitz von $1 \mathrm{Ctm}$. Breite angebracht. Fine Scheibe mit einem schwarzen und einem weissen Halbbreise wird auf jene Scheibe 
Nachdem so der eine der Exner'schen Beweise wohl erledigt sein dürfte, haben wir zu sehen, in wie weit die Beeinflussung des Eigenlichts und der Nachbilder durch einen continuirlichen, stärkeren mechanischen auf die Bulbi ausgeübten Druck etwa gegen die cerebrale Natur des Eigenlichts und der Nachbilder in Betracht kommen könnte.

Bei solchem Druck entwickelt sich bekanntlich aus dem Eigenlichte heraus das von Elliot, Purkynje und Aubert (1. c., S. 340) so überaus schön und genau geschilderte, licht- und farbenprächtige, wunderbar geformte und wechselvolle Feuerspiel, welches bei seinem, durch Verstärkung des Drueks żu beschleunigenden Ende in

gelegt und beide werden rotirt. Durch den Schilit, von einem bestimmten Punkte ans, beobachtet das Atge in einem Spiegel das Bild der Lichtmischung. Wenn etwa 24 Umdrehungen in der. Sekunde erreicht sind, so sieht man ohne Benutzung des Schlitzes gleichmässiges Grau, durch den Schlitz aber an einer bestimmten Stelle einen feststehenden Uhrzeiger-artigen schwarzen Strich, von dem aus nach beiden Seiten hin, znerst dunkel und immer heller wer dend, ein Gran sich bis zu weiss abschattirt. Jede andere Stelle des Schlitzes zeigt den schwarzen Uhrzeiger in einer anderen festen Zifferblattstellung. Verschiebt man den Schlitz um $180^{\circ}$ gegen die schwarz-weisse Scheibe, so bekommt man, an demselben Punkte des Apparats stehend, das Negativ - Bild des vorhergesehenen, sodass sich beide Bilder zu jenem gleichmässigen Grau ergänzen würden. Besonderes sieht man - die Erklärung liegt ja überall anf der Hand - bei einer schwarzen mit acht schmalen weissen Sectoren versehenen Scheibe. Bei zunehmender RotationsGeschwindigkeit entwickelt sich ein achtstrahliger schwarzer Stern, der still steht, sobald mit etwa 6-7 Umdrehungen $p$. Sec, das frei beobachtete Scheibenfeld eben nicht mehr flimmert. Bei etwas geringerer Umdrehungs-Geschwindigkeit dreht sich der Stern langsam in der Richtung wie die Scheibe und bei etwas grösser werdender Umdrehungszahl der Scheiben. fângt er an, sich in entgegengesetzter Richtung zu drehen und unter Abblassen sich in ein gleichmässiges Grau aufzulösen (bei doppelter Intermittenzzahl ist das Grau auch für das im Schlitz befindliche Auge gleichmässig). Das Stillstehen des achtstrahligen Sterns ist ein ziemlich empindlicher Index für die richtige Intermittenzzahl. 
einem ron Lichtstaub freien Schwarz nur kleine Fenerwerksreste zurücklässt.

Darüber, dass jenes Fener- und Farbenspiel in der (gedrücJten und anämisirten) Retina seinen Ursprung nimmt, kann ja kein Zweifel sein. Ebenso ist zweifellos während und nach Ablauf dieser retinalen Erscheinung der Lichtstaub aus dem Gesichtsfelde zunächst verschwunden, was für retinale Natur des Lichtstaubes zu sprechen scheinen könnte. Aber das gleiche Verschwinden des Lichtstaubes sehen wir auch, wenn die Retina durch ein objectives glänzendes Fenerwerk erregt wird; und ebenso fehlt fïr einige Zeit der Lichtstaub nach Ablauf eines länger datternden stärkeren objectiven Lichteindrucks (im Nachbilds-, resp. im Blendungsgebiete). So haben wir ja auch gesehen, wie ein lichtstaubfreies Sohwarz an jener Stelle des lichtchaotischen Gesichtsfeldes auftritt, von welcher bei Starrblindheit ein weisser Gegenstand entfernt wurde. Die Reaction des Eigenlichts auf Druck ist also im Principe keine andere, als auf objectives licht. Gesetzt nun, es wäre erwiesen, dass das Eigenlicht ausschliesslich im Hirn entsteht: natürlich würden wir es ,im Gesichtsfelde" sehen; dort sehen - wir aber auch die von der Retina her veranlassten Empfindungen. Jede solche in Folge objectiven Lichts oder mechanischen Drucks entstandene stärkere Erregung and Empfindung würde im Gesichtsfelde das Eigenlicht zum Verschwinden bringen müssen - theils durch Uebertönung, theils durch eine für den Willen unüberwindliche Ablenkung unserer Aufmerksamkeit; und nach dem Erlöschen derartiger stärkerer Lichtempfindung müsste, nach Weiss, assimilatorisch Schwarz zunächst zurückbleiben. Lässt sich dies so aus der Voraussetzung cerebraler Natur des Eigenlichts deduciren, so kann die Beeinflussung des Eigenlichts durch Druck und objeetives licht auch nichts gegen seine cerebrale Natur beweisen. (Das gleiche gilt selbstverständlich 
bezüglich der Abkürzung der Nachbilder durch Druck auf den Bulbus). Dagegen habe ich an mir eine besondere Beobachtung über die subjectiven Druck-Erscheinungen gemacht, welche eher gegen die retinale Natur des Lichtstaubes und gegen die Annahme sprechen könnte, dass das retinale Druck-Phänomen eine einfache Modification des Eigenlichts sei. (Leider babe ich die Druckversuche erst begonnen, als meine Scotome sich bereits zurückbildeten und keine absolut blinde Stelle mehr im gemeinsamen Gesichtsfelde war.) Ich sah die Stellen des Scotoms lange Zeit nach Beginn des Druckes am Feuerspiele gänzlich unbetheiligt und im Besitze ihres normalen (in neuerer Zeit gegen die Norm immer ausgesprochen verstärkten) Eigenlichtes verhleibend, während rings herum das prachtvolle Bild sich abspielte. Erst nach längerem Drucke betheiligte sich auch diese Partie. Uebrigens bekam sie nachher kein lichtstaubfreies Schwarz oder es stellte sich doch der Lichtstaub noch vor Beendigung des Versuchs im Scotom theilweise wieder her. Aber auch diese Thatsache verträgt sich zur Noth noch mit der Annahme retinaler Entstehung des Lichtstaubes. Ein wirklicher Beweis seiner retinalen Natur liegt indess jedenfalls überhaupt nicht vor, auch kaum ein rechtes Indicium, während ich eine Reihe von dringendsten Indicien, vielleicht sogar von Beweisen, für die cerebrale Entstehung ron Lichtstaub; Nachbildern und Tabaksscotom beigebracht zu haben glaube.

Anhangsweise seien einige Daten des Krankheitsverlaufes, sowie therapeutische und diätetische Erfahrungen mitgetheilt.

Die Affection begann nicht schleichend: am Vormittage des 28. September 1884 ( 40 Jahre ait), nahm ich im Sehen etwas wahr, was ich für das Nahen eines Flimmerscotoms hielt, wie ich solches schon etwa 5 Mal seit 12 Jahren. gehabt habe. Schon in den ersten Tagen mit Wahrscheinlich- 
keit, nach etwa 3-4 Wochen gleichmässiger Zunahme (ich war auf der Reise) konnte ich mit Sicherheit mir selber die Diagnose stellen, da ich die von Förster beschriebenen paracentrischen elliptischen Scotome nach aussen za projiciren vermochte nnd längere Zeit vorher sehr schweren Tabak geraucht hatte. Der Ausbruch der Affection erfolgte inmitten besonders guter Gesundbeit, Namentlich war mein Appetit,

Figur 1.
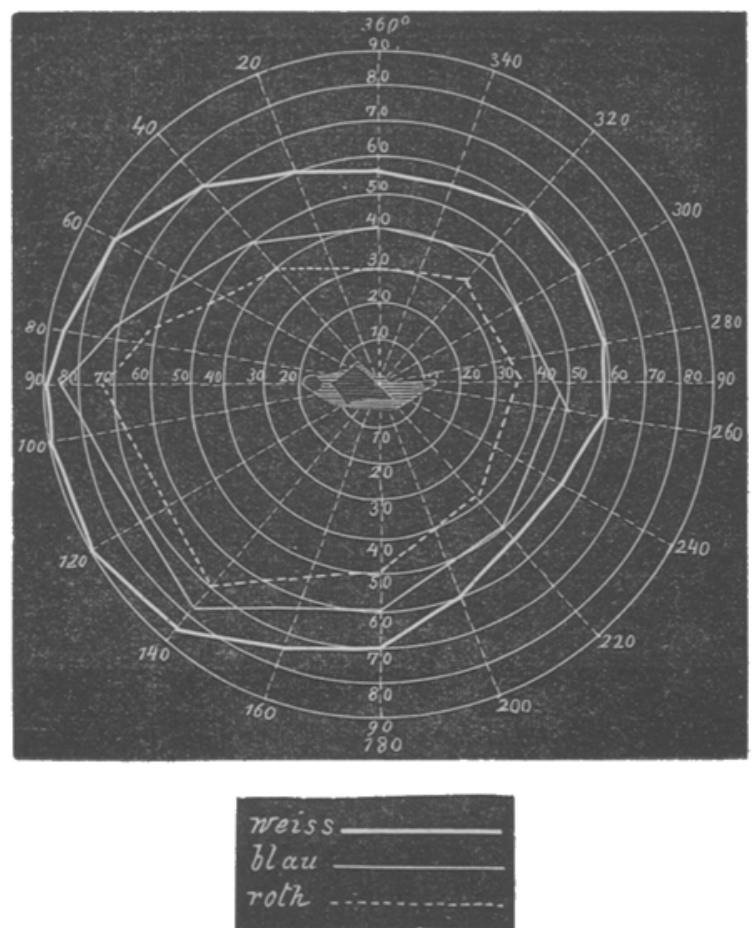

Schlaf und Ernährungszustand ganz vortrefflich. Ich hatte die letzten $1^{1 / 2}$ Jahre fast ausschliesslich (möglichst frische) österreichische Virginia-Cigarren (5 bis 7, manchmal wohl, aber selten, mehr, pro die, daneben gelegentlich auch Havannah) geraucht, mich aber in dieser Zeit sehr gut befunden. Alle die von Förster (1. c.) und Hirschberg (l. c.) angegebenen 
äusseren Bedingungen oder innern Störungen fehlten. Auch seitens des Herzens war nichts zu bemerken. Es bestand eine gewisse Reizung zu Durchfällen. Sobald ich die Diagnose gestellt hatte, rauchte ich nur noch unbedeutend, 1 bis höchstens 3 Cigarren, meist Havannah, täglich; trotzdem verschlimmerte sich der Zustand schnell; deshalb seit dem 5. December absolute Tabalis-Abstinenz; nichtsdestoweniger

\section{Figur 2.}
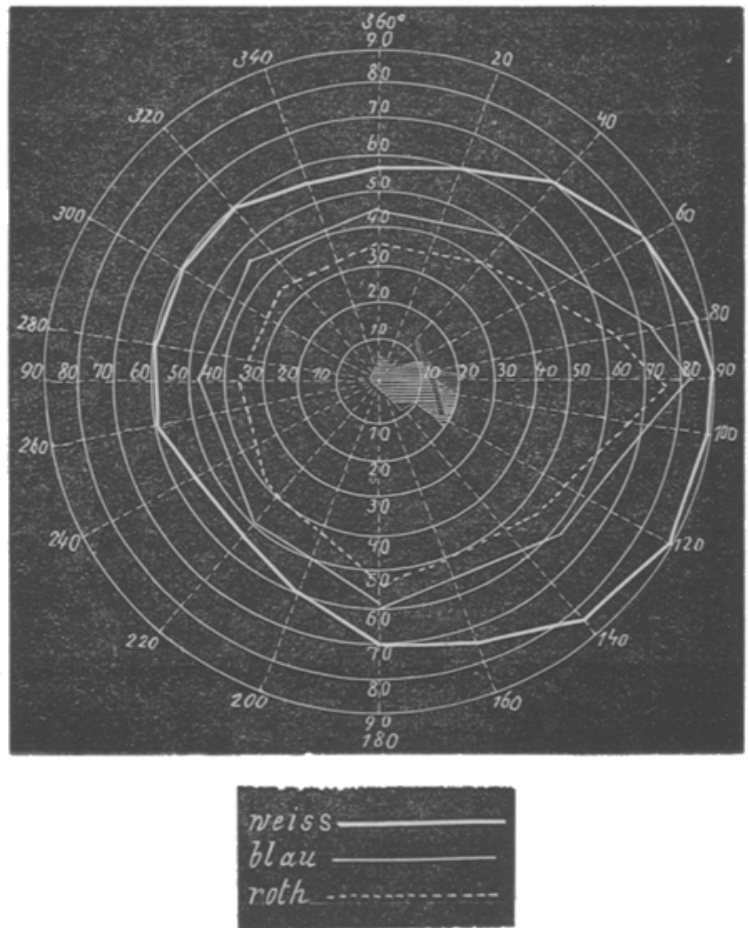

weitere Verschlimmerung während weiterer vier Wochen; von da an beginnende Besserung. Die Erkennbarkeit des Anfangs, die Verschlimmerung, trotz Aufhören der Giftzufuhr, sind anderwärts bisher nicht beobachtet worden: sie charakterisiren diese Vergiftungserscheinung als eine echte CumulationsWirkung dieses so sehr gewöhnbaren Giftes. 
In der orsten Zeit und übrigens schon vor dem Beginne fer Affection belästigte mich eine ungemein starko Disposition zu negativen Nachbildern und Blendungsseotomen (was übrigens auch für das nahe Beieinanderliegen der Nachbilder-Sphäre und des Sitzes der Affection spricht).

Des Experiments wegen wurde ein Mal (während des Rückganges) eine (Havannah-)Cigarre geraucht, um eine etwaige Analogie zur chronischen Morphin- und Alkobolvergiftung zu beachten; fïr eine bis zwei Stunden trat eine zwar bescheidene, aber subjectiv unzweifelhafte und auch am Perimeter nachweisbare Besserung des Sehvermögens in und um den (damals bereits nicht melir absolut scomatösen) Fixirpunkt auf, welcher dann aber eine deatliche Verschlechtung folgte.

Ebenso wirkte fur die gleiche Zeit $3 / 4$ Liter Wein oder $1 / 2$ Liter Bier, zumal bei gleichzeitigem Essen, während eine Mahlzeit ohne Altohol keine deutliche Besserung veranlasste. Jo mehr Alkohol, um so stärker und um so andauernder die nachfolgende Verschlechterung. Subcutane Injection von wenigstens 5 Milligramm Strychninum nitricum, gleichriel ob an der Schläfe oder am Beine, besserte intensiver und bis zu 24 Stunden, aber auch hier folgte eine evidente Versohlechterung.

Die für die Diatetik zu riehenden Schlüsse liogen auf der Hand. Es sei darauf hingedeutet, wie auch diese Erfahrungen für einen cerebralen Sitz des Tabaks-Scotoms sprechen.

Ein Mal auf der Höhe der Affection (December 1884) und ein Mal wahrend des Rutelganges (April 1885) hatte ich Anfälle ron Flimmer-Scotomen. Beim zweiten Falle begann das Flimmer-Scotom genau an der Grenze des Tabaks-Scotoms und verbreitete sich ron dort aus nach der Peripherie fast über das ganze Gesichtsfeld, während das Tabaks-Scoton auch in fast ganz wiederhergestellten Partien frei blieb.

Erwähnenswerth ist noch, dass im Stadium ausgesprochensten Rückganges der Erkrankung und begonnener Verkleinerung der Scotome, trotz absoluter Tabak-Abstinenz, im dritten bis vierten Monate bei woehenlang liegendem Schnee und ankaltendem somnigen Frostwetter (Januar 1885), eine älusserst störende Asthenopie anftrat, welche beim Versuche zal lesen oder zu schreiben schon nach wenigen Minuten alle Buch- 
staben verschwinden liess. Consequenter Gebrauch indifferent grauer Brille dunkler Nuance beseitigte diesen Zustand, ein deutlicher Wink, dass der Gebrauch dunkler Brillen überhaupt zur Unterstützung der Heilung derartiger Intoxicationsamblyopie von wesentlicher Bedeutung sein dürfte.

Ich lasse die von Herrn Dr. L. Rosenmeyer, erstem Assistenten der von Herrn Collegen Sattler geleiteten ophthalmologischen Klinik gemachten Aufzeichnungen wörtlich folgen und bemerke, dass die erste Aufnahme erst im vierten Monat bei bereits begonnenem Rückgang der Affection, zur Zeit der erwähnten Asthenopie am 29. Januar 1885 erfolgte:

„Links: früher $S=6 / 5$, jetzt $=6 / 86$.

Rechts: früher $S=6 / 18$, jetzt $=6 / 60$.

Die Gesichtsfeldaufnalme ergiebt beiderseits normale Aussengrenzen für weiss, blau und roth, ebenso normalen peripheren Lichtsinn, Objecte ron $2 \mathrm{Mm}$. Grösse rerschwinden, um den Fixirpunkt in der aus Tafel 1 ersichtlichen Ausdehnung.

Hierauf allmälige Besserung, die weissen und farbigen Objecte erscheinen stellenweisse im Scotom bei den in der Zwischenzeit vorgenommen Tntersuchungen.

Die Aufnahme am 18. März 1885 ergiebt folgenden Status:

Links: $S=6 / 18$ Jäger 3 wird leicht gelesen. cfm. für roth $10 / 15^{*}$ ), für blau $9 / 15$.

cL herabgesetzt bei N. ${ }^{13} / 15$ wird roth $5 / 15$ (statt $8 / 15$ ). $", 9 / 15, \quad, \quad 1 / 15$ in 1 Meter Entfernung (statt $5 / 15$ in 4 Meter Entfernung erkannt). p.L. normal.

Das Scotom nach inuen und oben rom Tixirpunkte geschwunden mit Ausnalme einer kleinen Stelle im H. IN zwischen $11-13^{0}$ nach innen vom Fixirpunkte, wo die Objecte gänzlich verschwinden.

Rechts: $\mathrm{S}=6 / 36$ Jäger 14 mit Mühe, cfm. für roth $7 / 15$;

*) Die Zahlen beziehen sich auf den Apparat zur Lichtsinnprüfung ron Dr. L. Wolffberg, cf. dieses Archiv, Band XXXI, 1, S. 1. 
für blau $6 \% 15$. Aussengrenzen vom Scotom beinahe unverändert, Fixirpunkt nicht frei, im Scotom stellenweise hellere Partien sowohl für weiss, als farbige Objecte.

Die vergleichende Aufnahme vom 22. April 1885 ergiebt wenig nennenswerthe Veränderungen.

Die dunkel schraffirten Stellen im Scotom zeigen die Grenzen derselben im linken Auge bei der letzten Aufnahme.

Nachschrift, 27. Juni 1885:

Inzwischen wesentliche Besserung; Scotomgrenzen ganz verwischt; innerhalb der alten Grenzen nirgends mehr absolutes Scotom, nur für blau noch einige ganz unempfindliche Stellen, sonst nur noch leicht amblyopische Stellen.

L. $\mathrm{S}^{5} / 6$. cfm. für roth $11 / 15$; für blan und grün $12 / 15$. cL bei $H^{11} / 15$. cfm. roth $1 / 15$; blau und grün $5 / 15$ (?). Also bei beinahe wieder restituirter Sehschärfe centraler Lichtsinn noch herabgesetzt. 